\title{
Tailored Nanopatterning by Controlled Continuous Nanoinscribing with Tunable Shape, Depth, and Dimension
}

Dong Kyo Oh, ${ }^{a \neq}$ Seungjo Lee, ${ }^{a \neq}$ Seung Hu Lee, ${ }^{b \neq}$ Wonseok Lee, ${ }^{a}$ Gyubeom Yeon, ${ }^{a}$ Nayeong Lee Kang-Soo Han, ${ }^{d}$ Sunmin Jung, ${ }^{a}$ Dong Ha Kim, ${ }^{a}$ Dae-Young Lee, ${ }^{d}$ Sang Hoon Lee, ${ }^{a c}$ Hui Joon Park, e* $^{*}$ and Jong G. Ok ${ }^{a^{*}}$

${ }^{a}$ Department of Mechanical and Automotive Engineering, Seoul National University of Science and Technology, Seoul 01811, Korea

*E-mail: jgok@seoultech.ac.kr

${ }^{\mathrm{b}}$ Department of Energy Systems Research, Ajou University, Suwon 16499, Korea

${ }^{c}$ Research Center for Electrical and Information Technology, Seoul National University of Science and Technology, Seoul 01811, Korea

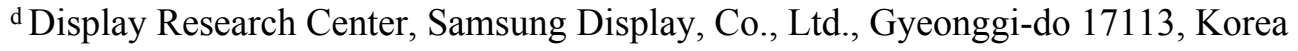

${ }^{\mathrm{e}}$ Department of Organic and Nano Engineering, Hanyang University, Seoul 04763, Korea

*E-mail: huijoon@hanyang.ac.kr

These authors contributed equally to this work.
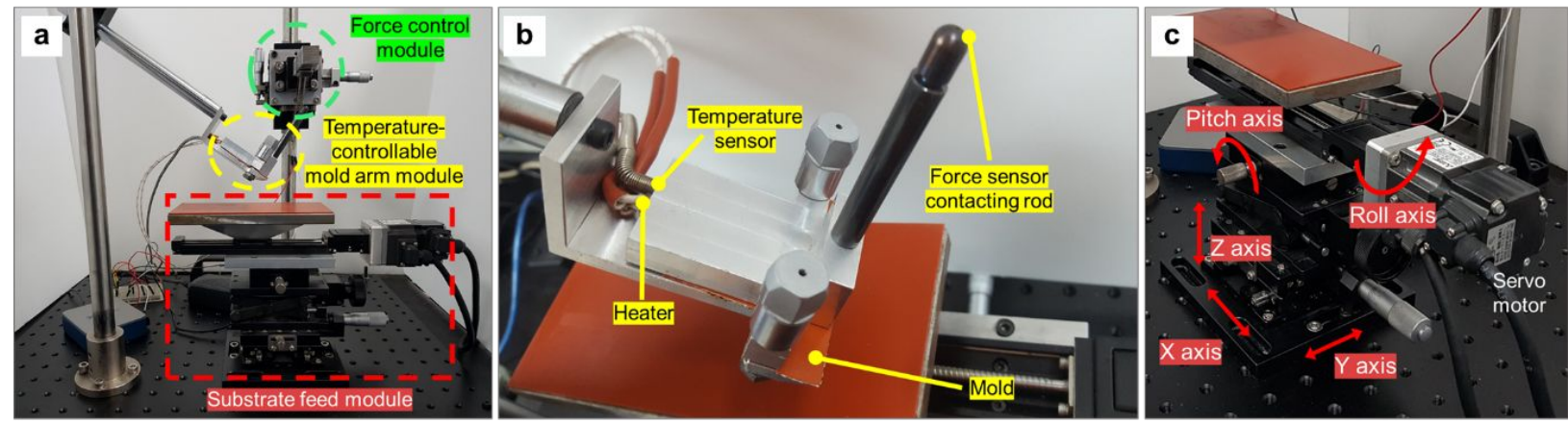

Figure S1. (a) Assembly-completed DNI processing system, comprising three main modules: force control module, temperature-controllable mold arm module, and motorized substrate feed module. Detailed views of (b) temperature-controlled mold arm module and (c) substrate feed module. 


\section{a Traditional Extrusion}

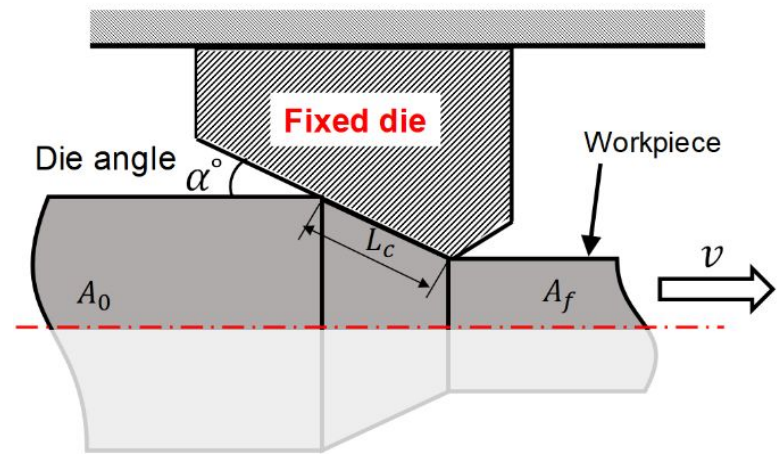

b DNI

Variable force, mold angle, and temperature

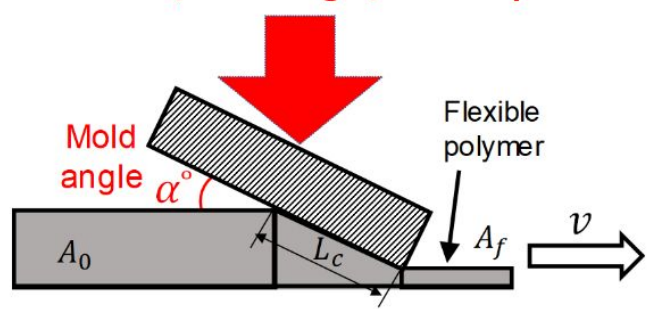

Figure S2. Schematic comparison between (a) traditional extrusion and (b) DNI. These two processes are analogous to each other in terms that they both induce plastic deformation of materials by pushing them out through the openings that are smaller than the materials' original size. The final deformed workpiece is mainly determined by the fixed die angle in traditional extrusion (a), while the final nanopattern profile can be tailored by controlling the normal applied force, mold mounting angle, and temperature in DNI (b).
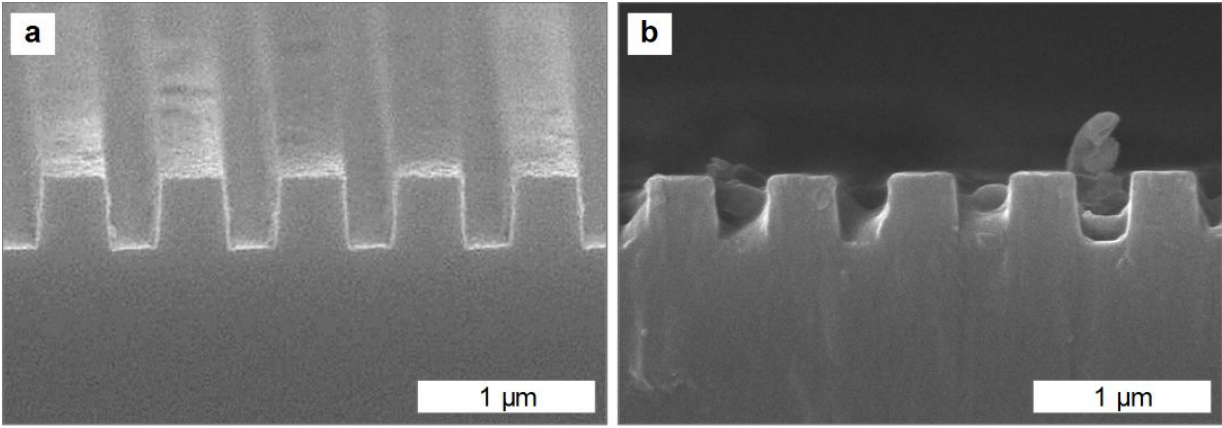

Figure S3. SEM images of (a) the as-cleaved $700 \mathrm{~nm}$-period $\mathrm{SiO}_{2}$ nanograting mold and (b) identical one used for 30 times of $10 \mathrm{~cm}$-long DNI strokes. The overall mold outline shape remains intact, but a slight amount of surrounding dust is piled in the grating valleys of the used mold (b). While this is not critically affecting the final nanopattern profile, the mold can be periodically recleaved for defect-free long stroke patterning. 


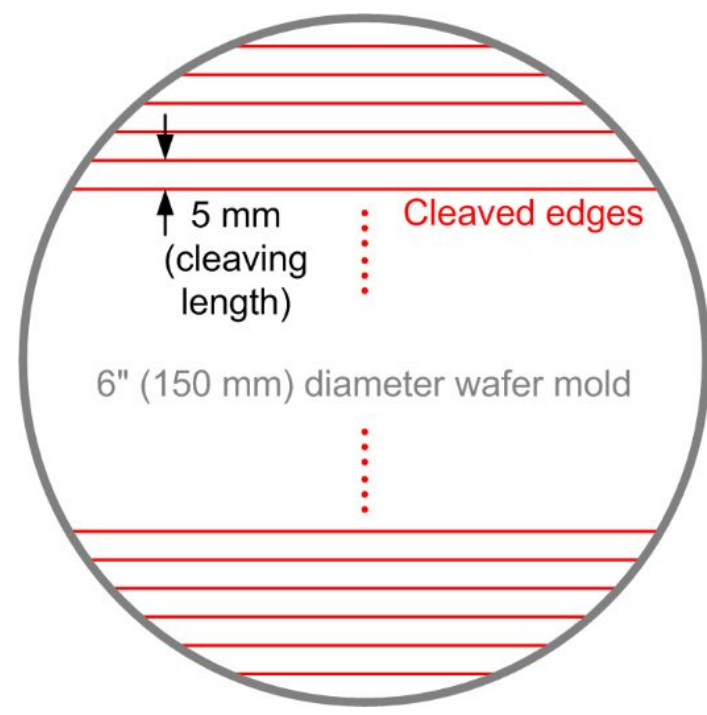

(1) Original wafer mold area $=17,671 \mathrm{~mm}^{2}$

(2) Total length of cleaved edges $=3,511 \mathrm{~mm}$ (summation of all red lines)

(3) Reliable DNI stroke per edge $=500 \mathrm{~mm}$

(4) Total DNI-able area $=(2) \times(3)=1,755,760 \mathrm{~mm}^{2}$

(5) Processable area ratio $=(4) /(1)=99.35$

Figure S4. Simplified quantitative evaluation of DNI productivity by comparing the original 6"diameter wafer mold area and total DNI-processable area. Considering that a distance of $5 \mathrm{~mm}$ between the edges is desired for handy cleaving, we can obtain the total length of cleaved edges of $\sim 3.5 \times 10^{3} \mathrm{~mm}$ per 6 " wafer. By multiplying it by the statistically averaged stroke length of 500 $\mathrm{mm}$ for reliable DNI, we can get the total DNI-processable area of $\sim 1.75 \times 10^{6} \mathrm{~mm}^{2}$. This is $\sim 100$ times larger than the original 6" wafer mold area. 